\section{An APL permutation routine for computer search operations}

\author{
JOHN C. SCHMITT \\ State University of New York \\ Binghamton, New York 13901
}

As Pecter (1975) has pointed out, one problem associated with computer searches for maximization, goodness of fit, etc. is the limited availability of permutation routines, particularly in convenient languages. If a single ordering of $\mathbf{N}$ integers produces an optimal combination (according to whatever criteria), the problem may be stated as that of creating a list of all N! strings to be searched. This is an enormous task for any $\mathrm{N}$ greater than, say, 4 or 5 , and may be a particularly noxious one for researchers limited to APL dial-up facilities rather than local computer support. Function PERMUTATIONS (see Table 1) is executed with the argument SIZE (for $\mathrm{N}$ ) and calls EXTEND, which contains the algorithm for adding an additional integer to a string of any length. EXTEND may also be used independently for that purpose. The final solution, an $\mathrm{N}$ ! by $\mathrm{N}$ matrix named PERMS, whose rows represent all permutations of the integers 1 to $N$, is left global to the workspace and available for any searching functions that are performable in APL.

Support and Availability. These functions were developed on an IBM 370/128 system, under APL/SV. They are extensively condensed (and, hence, cryptic) for efficiency in light of the large number of line execu-
Table 1

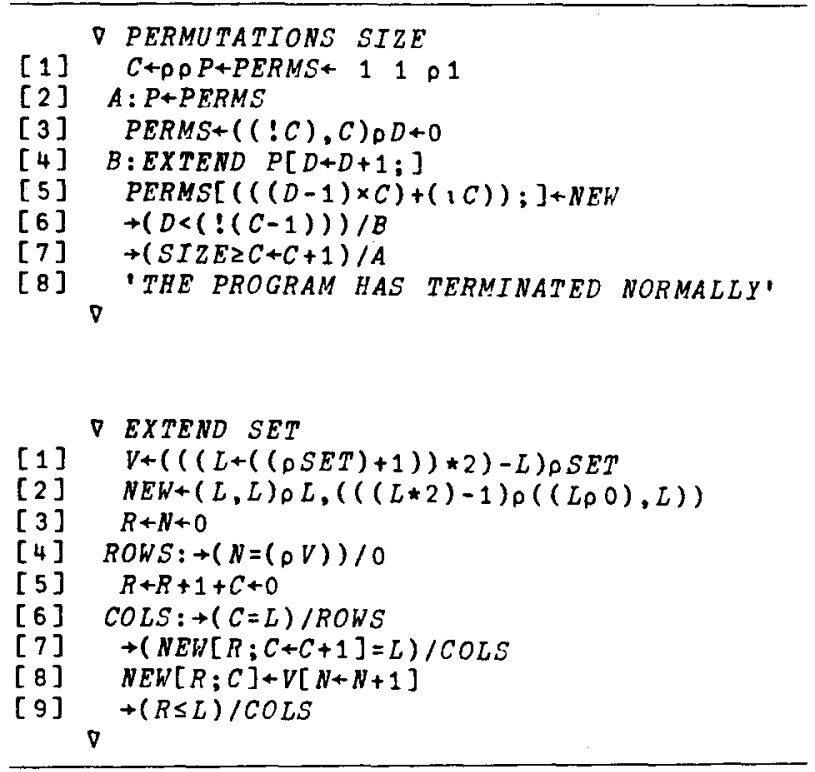

tions involved. Line-by-line comments, copies, and example uses may be obtained at no cost from the author at the Department of Psychology, State University of New York, Binghamton, New York 13901.

\section{REFERENCE}

Pecter, R. Subroutine for generating all permutations of $\mathbf{N}$ integers. Behavior Research Methods \& Instrumentation, $1975,7,57$. 